\title{
A Method to Implement Data Extraction Using and Optical Gate Based on SOR - NOLM
}

\author{
Yalin Guan, Ruidong Wang, Xinqiao Chen, Zhen Zhang, Zhonghang Duan, Cuizhu Chen \\ College of Information Engineering, Communication University of China, Beijing 100024, China \\ 123495518@qq.com
}

\begin{abstract}
A method was proposed to implement data extraction using AND optical gate operation on semiconductor optical amplifier (SOA) based nonlinear optical loop mirror (NOLM). The optical data extraction in FSK/ASK orthogonal modulation system was achieved with the proposed AND gates, in order to prove its feasibility. Through the simulation, the data extraction in orthogonal modulation system with a 10Gbit/s NRZ pseudo-random payload signal and $2.5 \mathrm{Gbit} / \mathrm{s}$ label signal was demonstrated.

Index Terms - Optical label, optical logic gate, orthogonal modulation, semiconductor optical amplifier.
\end{abstract}

\section{I . Introduction}

Optical swapping and optical computing are the core unit to achieve all optical signals processing in future all optical networks, they all based on optical logic gates, which are core devices to implement optical swapping system and the key factor of decision network performance. All optical logic gates(XOR - AND)are indispensable part for all optical signal processing, which can be used in all-optical clock recovery, alloptical demultiplexing, all-optical data extraction, etc. Therefore, it has great application potential in future all optical high speed communication network and new generation optical computing.

At present, the research of all optical logic gates are focused on the using of nonlinear effect of optical fiber and semiconductor optical amplifier. The nonlinear effect of optical fiber is weak, so that the logic gates based on nonlinear effect of optical fiber will achieve idea effect only by high power input signal or long fiber. SOA become the main functional device to implement all kinds of optical logic gates, because it has many advantages such as small in size, easy to integrate, short response time and strong nonlinear effect. Taking the nonlinear effect advantage of SOA can realize many kinds of optical logic gates, but the design scheme to realize AND gate is relatively less, for example, using twolevel SOA's cross gain modulation effect implementing AND gate, using Mach-Zehnder interferometer based on SOA implementing AND gate, etc. optical data extraction is an important part in optical label swapping system, it is the key factor for all optical network. At present, there have been many design and experiment scheme about optical data extraction. These schemes have it own advantages, but they also have some disadvantages. Article [1] realized all optical data extraction by using start bit in data packet generating address. This method has the advantage of easy to realize and doesn't need complicated clock recovery module; but the realization of control pulse division module is closely related to the length of the packet address, it is needed to increase or decrease the same number of delay fiber when the length of address is increased or decreased. Article [2] realize data extraction from packet through the switch module which are consisted of Mach-Zehnder interferometer based on two-level SOA and Fabry-Perot filter, clock recovery module and address-data separation module; this scheme has the advantage of high speed and flexible; but configuration structure is too complex. By contrast, the method to implement data extraction using AND optical gate has the advantage of high speed in data extraction, simple structure and easy to integrate. In this article we put forward a method to implement AND optical gate using two SOAs based NOLM structure, and in order to prove the AND gate having the ability in information processing, the AND gate is applied to load FSK/ label ASK orthogonal modulation system to extract data information. Through the simulation, the data extraction in orthogonal modulation system with a 10Gbit/s NRZ pseudo-random payload signal and $2.5 \mathrm{Gbit} / \mathrm{s}$ label signal is demonstrated.

\section{II . Operation Principle}

The principle to implement AND gate operation on SOA based NOLM which also known as a nonlinear Signac interferometer was shown in Fig.1. In this structure, SOA is placed slightly asymmetrical, so that a data signal which is split to $\mathrm{CW}$ and $\mathrm{CCW}$ components, passed the SOA at different times. After a complete circulation in the loop The $\mathrm{CW}$ and $\mathrm{CCW}$ signal interfere against at optical coupler. Without control pulse injected into the loop, both $\mathrm{CW}$ and CCW signal will experience destructive interference since both signals are amplified linearly in non-saturate SOA regime. The nonlinear effect will happen if a control pulse injected into the loop. The control pulse is calculated well so that it will reach SOA in between $\mathrm{CW}$ and $\mathrm{CCW}$ signal pulse. Because of the CCW pulse passes the SOA slightly after the control pulse, it will get saturated SOA regime. The gain and phase change for $\mathrm{CW}$ and $\mathrm{CCW}$ are not the same. The reason is that $\mathrm{CW}$ pulse reaches the SOA before the control pulse and experiences a constant gain and phase shift, but for the $\mathrm{CCW}$ pulse the gain and phase shift is changing during the propagation through SOA. The distance of the SOA $\Delta x$, from the centre of loop determine the switching windows. So the switching windows can be changed by using delay time (DL). When the control pulse passes through the SOA between $\mathrm{CW}$ and $\mathrm{CCW}$ pulse, only the last is affected by a phase change because of the saturation of SOA. So ,if the phase change between the pulses 
is $\pi$, then the two pulses interfere constructively at the coupler. If they are in phase, there will be an output pulse; if they are out of phase, there will be no output[3].

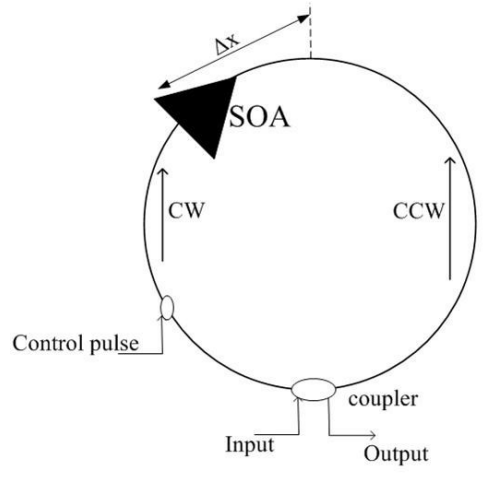

Fig.1 The SOA-NOLM diagram

It is shown in Fig.2. We designed a NOLM structure in this way by using two SOA. Thus configuration has the same behaviors as depicted in Fig.1. To implement AND gate by SOA-NOLM two SOA are used in the loop mirror to perform light propagation in clock wise and counter clock wise.

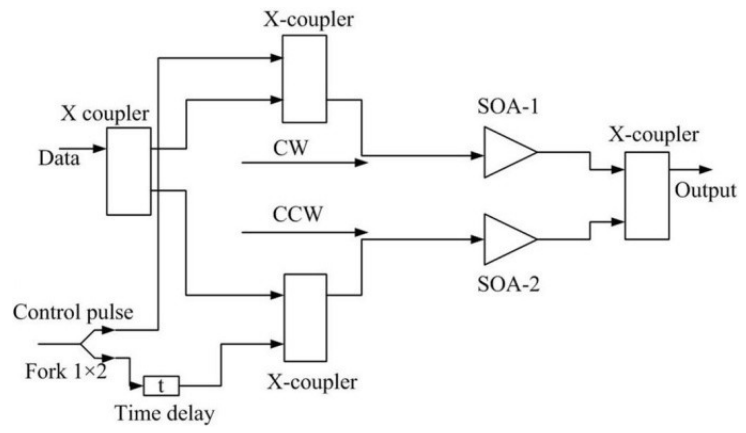

Fig.2 The AND gate opreation on two SOA based NOLM

The principle to implement optical data extraction using AND gate was depicted in Fig.3. To extract the desired bits from label information, the label information sequence which was needed to define was "1100". By asserting 1st and 3rd were the data needed extraction from label information, in order to achieve it, 1st and 3rd of control information which decides desired bit position were set as " 1 ", other bits were set as " 0 ", and it meant that pulse sequence of control information was "1010". Through the logical operation of AND gate 1st and 3 rd of output sequence were the extraction data which we needed.

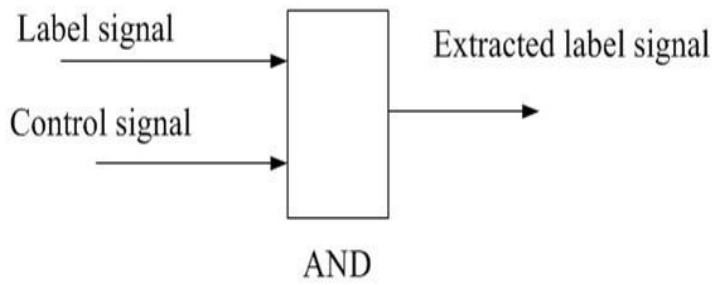

Fig.3 The data eatraction using AND gate

\section{III . Experiment Demonstration}

The FSK/ASK orthogonal modulation optical data extraction system structure of simulation was depicted in Fig.4. There were two parts in AND gate optical extraction system. At transmitter, the continuous-wave source was a tunable laser operating at $1550 \mathrm{~nm}$. A pulse generator created a $10 \mathrm{GHz}$ NRZ pseudo-random pulse train, which was synchronously frequence-modulated by $10 \mathrm{Gbit} / \mathrm{s}$ payload data through a frequence modulator to achieve frequence modulation. a Mach-Zehnder modulator (MZM) was used to insert label information at $2.5 \mathrm{Gbit} / \mathrm{s}$ signal by intensity modulation. The pulse sequence of label was: 1100. The generated FSK/ASK packet was transmitted $80 \mathrm{~km}$ in standard signal-mode fiber (SSMF) and $16 \mathrm{~km}$ dispersion - compensating fiber (DCF). At data extraction part, using the AND gate based on SOANOLM to extraction optical data, the control information pulse sequence which was also intensity modulated by MZM injected in SOA set as "1010". In the end, the extracted data was filtered by a Fiber Bragg Grating (FBG) filter and switched by a Photo Detector [4].

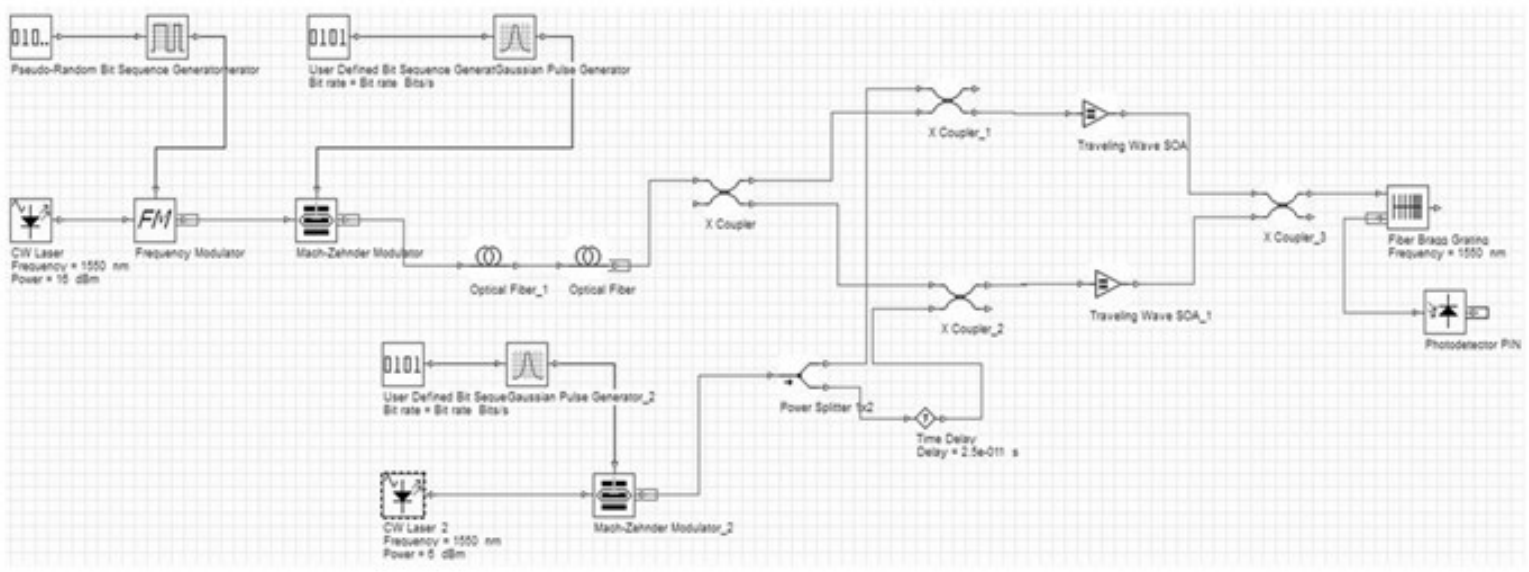

Fig.4 FSK/ASK orthogonal modulation data extraction system 


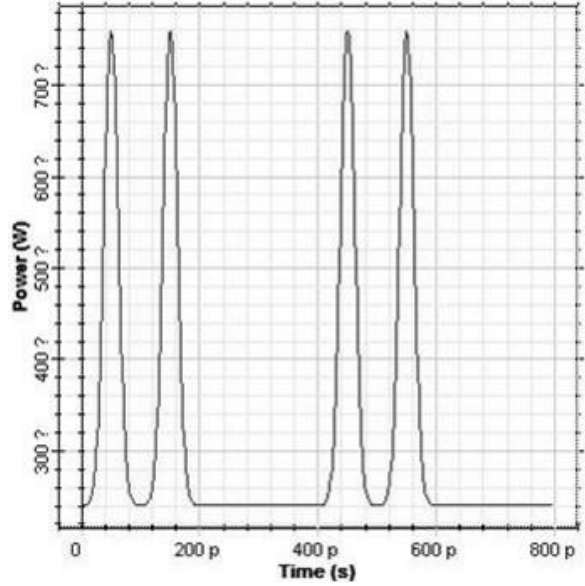

(a) label information

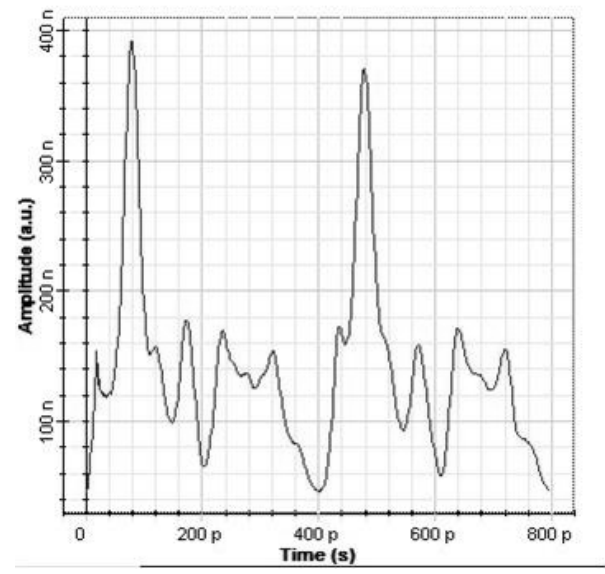

(b) extracted data information

Fig.5 Time domain waveform of label information

Time domain waveform of label information after data extraction by AND optical gate based on SOA-NOLM was shown in Fig.5. The label information was depicted in Fig.5 (a). The extracted data information was depicted in Fig.5 (b). From the extracted data information waveform, pulse information of the 1st bit was: "1", the 3 rd bit was " 0 ", consistent with the theoretical value "1000". Therefore, the method to extraction data information using the AND optical gate based on SOA-NOLM was feasible.

\section{IV . Conclusion}

By using the SOA-NOLM interfere structure, all optical logic AND gate was realized. The AND gate was applied to load FSK/ label ASK orthogonal modulation system to extract data information. In the simulation experiment, the label information was extracted. Optical data extraction is a way of extraction and recognition optical label in optical domain, and it has extensive application prospect in high speed all-optical networks. The method of this optical data extraction based on optical logic AND gate has high speed of optical data extraction and simple structure.

\section{References}

[1] G.L. Papadimitriou. Papazoglou and A.S. Pomportsis, Optical Switching: Switch Fabrics, Techniques, and Architectures,IEEE J. Lightwave Technol, 2003,21(2): 384-405.

[2] Jourdan,D. Chiaroni,E. Dotaro,et al.,The Perspective of Optical Packet Switching in IP-Dominant Backbone and Metropolitan Networks,IEEE Commun. Mag.,2001,39(3):136-141.

[3] Rosnahlizawati Rostam, Mohamad Halim Abd. Wahid, Mohd Farizal Faridus, et al. The Effect of Siganl Repetition Rate, Injected Current and Swithching Windows on Cross-Phase Modulation in SOA-NOLM, RSM2011 Proc., 2011,Kota Kinabalu, Malaysia.

[4] Nan Chi, Jianfeng Zhang, Pablo V. Holm-Nielsen, et al. Transmission and Transparent Wavelength Conversion of an Optically Labeled Signal Using ASK/DPSK Orthogonal Modulation.IEEE PHOTONICS TECHNOLOGY LETTERS, VOL. 15, NO. 5, MAY 2003:760-762. 